\title{
Response of Onion Yield and Quality To Different Planting Date, Methods and Density
}

\author{
S.H. Abou Khadrah, A.A.El-Sayed, L.S.M.Geries*\# and W.K.L. Abdelmasieh* \\ Agron. Department., Faculty of Agriculture, Kafrelsheikh Univ.; Kafrelsheikh and \\ *Onion Research Department, Field Crops Research Institure, Agricultural Research \\ Center(ARC), Giza, Egypt.
}

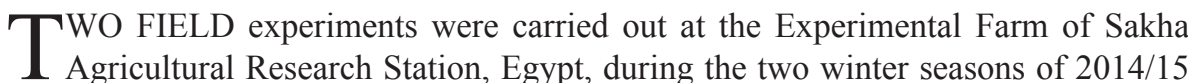
and 2015/16. The experiments were conducted to find out the suitable transplanting date $\left(15^{\text {th }}\right.$ December, $1^{\text {st }}$ January and $15^{\text {th }}$ January), the best planting method (ridges and wide furrows) and to assess three plant densities i.e.,30, 45 and 60 plants $/ \mathrm{m}^{2}$ for vegetative growth, yield and quality of onion under North Delta conditions. The main results showed that, the vegetative growth behavior was improved, also yield and its components and quality of onion were increased by transplanting onions on $15^{\text {th }}$ Dec. date in both seasons. Wide furrows planting method resulted in a significant increase in the most of vegetative growth characteristics, as well as total bulb yield and its components and bulb quality. Increasing plant density up to 45 plants $/ \mathrm{m}^{2}$ significantly increased most vegetative growth characteristics, total and marketable bulbs yield/fad and bulb qualityin 2014/ 15 and 2015 /16 seasons.

Transplanting onions on $15^{\text {th }}$ Dec. in wide furrows planting system significantly recorded the tallest plants, leaf area/plant, total bulbs yield, marketable bulbs yield and average bulb weight.Onion cultivar transplanted at $15^{\text {th }}$ Dec. with 30 plants $/ \mathrm{m}^{2}$ resulted in a markedly increase in plant height, plant fresh and dry weight and leaf area/plant at the three sampling dates in both seasons. While, total and marketable bulbs yield/fad, was increased by transplanted at $15^{\text {th }}$ Dec.with 45 plants $/ \mathrm{m}^{2}$.The highest vegetative growth, total bulbs yield and bulb quality resulted from onions when were planted with 45 plants $/ \mathrm{m}^{2}$ in wide furrows planting method.

The economic evaluation showed that the highest gross and net return per fad and net benefit-cost ratio obtained from transplanting onions at $15^{\text {th }}$ Dec.(early date), wide furrows planting method with planting 45 plants $/ \mathrm{m}^{2}$. So, this study recommended onion farmers at North Delta of Egypt to transplanting onions at $15^{\text {th }}$ Dec. (early date) in wide furrows planting method with the plant density of 45 plants $/ \mathrm{m}^{2}$ to achieve the highest economic yield.

Keywords: Onion, Allium cepa L., Transplanting date, Planting method, Density.

\section{Introduction}

Onion (Allium cepa L.) belongs to the family Alliaceae genus Allium (Griffiths et al., 2002), and it is a cross-pollinated, herbaceous and biennial crop. It is one of the most important cool season vegetable crops. It ranks seconds among all vegetables in economic importance after tomatoes in the world (Malloret al., 2011).

Egypt occupies the fifth place among the ten countries in the world in terms of area onions, ranked ninth in terms of productivity. Egypt's production of onions reached in season 2013, approximately two million tons, cultivated onions space in Egypt is about $8 \%$ of the total cultivated area in the world cultivated area of the metal annually in Egypt about 150 thousand faddan (Annual report of the Statistics and Agricultural Economics Department, The Ministry of Agriculture, Egypt).

\#Corresponding author email: dr_labib_sobhy@yahoo.com 
Transplanting dates of onion seedlings reflecting the effect of edaphic factors and all environmental conditions in large scale on growth, bulb yield and bulb quality, which differ widely from region to another. Thus, optimum transplanting dates have a vital role in maximizing growth, bulb yield and its quality of onion (Bharti \& Ram, 2014; Misra et al., 2014 and Ali et al., 2016 b)

Cultivation method considers one of the effective means for rationalization of irrigation water. Wide furrows may allow saving irrigation water and still maintaining satisfactory levels of production. So, there is an urgent need to improve irrigation water management for local farmers (Farrag, 1995; Kanwar \& Akbar, 2013 and Geries et al., 2015).

Plant population is one of production factors that need to be optimized. The optimum use of spacing or plant population has dual advantage. It avoids strong competition between plants for growth factor such as water, nutrient and light. Also, optimum plant population enables efficient use of available crop land without wastage (Zubelidia \& Gases, 1977).

A variety of diseases and disorders affect onions and related crops, most of the major onion diseases are caused by fungi or bacteria, or sometimes by soil nematodes. Among these diseases the downy mildew and purple blotch of onion is one of the major constraints in onion cultivation. Downy mildew of onion, caused by the fungus Peronospora destructor Berk, is worldwide in occurrence and causes devastating economic losses to the crop (Cook, 1932; Viranyi, 1974 and Yadav et al., 2013). Yield reductions of onion bulbs due to downy mildew outbreaks may range from $30 \%$ up to $70 \%$ when the environment is conducive for the disease (Van Doorn, 1959; Maude, 1990, Raziq et al., 2008 and Pablo et al., 2011).

Also, purple blotch of onion caused by Alternaria porri Ell.,which an important disease of onions throughout the Egypt. Under favorable environmental conditions purple blotch can spread rapidly and yield losses can exceed 30 $\%$. High relative humidity (80 to 90\%) and optimum temperature $\left(24 \pm 1^{\circ} \mathrm{C}\right)$ are favour for further development of purple blotch disease and causing considerable yield losses. Shahanaz et al. (2007) reported losses about 50 to 100 per cent due to purple blotch disease. Downy mildew and purple blotch management includes the means such as planting timing, resistant cultivars, soil drainage, seed or bulb health stock, wind block, planting dates, planting method and plant density. (Hoffmann et al., 1996; Develash \& Sugha, 1997; Krauthausen et al., 2001; Schwartz \& Mohan, 2008 and Surviliene et al., 2008.)

Therefore, the main objective of this study is to investigate the optimal transplanting date $\left(15^{\text {th }}\right.$ December, $1^{\text {st }}$ and $15^{\text {th }}$ January), method (ridges and wide furrows) and density (30, 45 and 60 plants $/ \mathrm{m}^{2}$ ) for vegetative growth, yield and quality of Giza Red onion cultivar and the relationship with diseases development under North Delta conditions.

\section{Materials and Methods}

Two field experiments were laid out at the Experimental farm of Sakha Agricultural Research Station, Agricultural Research Center (ARC) during two successive winter seasons of 2014/15 and 2015/16. Mechanical and chemical analysis of the experimental sites in the first and second seasons according to Piper (1950) and Jackson (1967) are presented in Table 1. The averages of weather factors for Kafr El-Sheikh Governorate during the onion crop growing seasons are recorded in Table 2.

In both seasons, the seeds of Giza Red onion cultivar were sown in the nursery on $15^{\text {th }}$ October, $1^{\text {st }}$ and $15^{\text {th }}$ November, while transplanting took place after 50 to 60 days of sowing on $15^{\text {th }}$ December, $1^{\text {st }}$ and $15^{\text {th }}$ January in both seasons. Each experimental plot area was $12.6 \mathrm{~m}^{2}$ (included 6 ridges, $60 \mathrm{~cm}$ width and 3 wide furrows, 120 $\mathrm{cm}$ width with 3.5 meter long for each. A splitsplit plot design with four replicates was used in this study. The main plots were designated for the three planting dates $\left(15^{\text {th }}\right.$ December, $1^{\text {st }}$ January and $15^{\text {th }}$ January), whereas planting method: ridges "60 cm width" and wide furrows "120 cm width" were randomly distributed in sub - plots. Three plant densities of 30, 45 and 60 plants $/ \mathrm{m}^{2}$ were allocated in sub-sub-plots.

All the cultural operations like nursery raising, main field preparation, transplanting, fertilization, irrigation, weeding, plant protection etc. were laid out as per the recommendations in order to obtain a successful crop. 
TABLE 1. Mechanical and chemical analysis of the experimental soil samples at the depth of $(0-30 \mathrm{~cm})$ in $2014 / 15$ and 2015/16 seasons.

\begin{tabular}{lcc}
\hline \multirow{2}{*}{ Determination } & \multicolumn{2}{c}{ Season } \\
\cline { 2 - 3 } & $\mathbf{2 0 1 4 / 1 5}$ & $\mathbf{2 0 1 5} / \mathbf{1 6}$ \\
\hline Mechanical analysis: & \\
Sand \% & 17.88 & 17.64 \\
Silt \% & 32.89 & 33.52 \\
Clay \% & 49.23 & 48.84 \\
Textural class & Clay & Clay \\
Chemical analysis: & & \\
$\mathrm{PH}$ & 8.10 & 7.82 \\
$\mathrm{EC}$ (m.mhos/cm.) & 2.45 & 2.12 \\
Organic matter \% & 1.85 & 1.52 \\
CaCo3\% & 3.16 & 3.08 \\
Available N ppm & 18.30 & 27.8 \\
Available P ppm & 6.18 & 5.95 \\
Available K ppm & 279.35 & 296 \\
Cations (meq/L): & & \\
$\mathrm{Ca}$ & 3.5 & 5.8 \\
$\mathrm{Mg}$ & 2.9 & 3.4 \\
$\mathrm{Na}$ & 9.88 & 11.5 \\
$\mathrm{~K}$ & 0.40 & 0.58 \\
Anions (meq/L): & & \\
$\mathrm{CO}{ }_{3}^{-}$ & 0.00 & 0.10 \\
$\mathrm{HCO}_{3}^{-}$ & 4.00 & 3.46 \\
$\mathrm{Cl}^{-}$ & 5.60 & 4.30 \\
$\mathrm{SO}_{4}^{--}$ & 10.13 & 13.52 \\
\hline
\end{tabular}

TABLE 2. Monthly averages of weather factors for Kafr El-Sheikh Governorate during 2014/15 and 2015/16 seasons.

\begin{tabular}{lccccccc}
\hline \multirow{2}{*}{ Month } & Season & \multicolumn{3}{c}{ Temperature C } & \begin{tabular}{c} 
Relative \\
Humidity \\
\cline { 3 - 6 } \%
\end{tabular} & $\begin{array}{c}\text { Wind } \\
\text { speed } \\
\text { (km/24h) }\end{array}$ & $\begin{array}{c}\text { Class A pan } \\
\text { evaporation } \\
\text { (mm/day) }\end{array}$ \\
\hline \multirow{2}{*}{ December } & 2014 & 22.3 & 9.7 & 16.0 & 76 & 46.0 & 172.4 \\
January & 2015 & 20.4 & 8.4 & 14.4 & 78 & 57.9 & 248.7 \\
& 2015 & 18.8 & 6.5 & 12.6 & 75 & 70.8 & 270.9 \\
February & 2016 & 18.4 & 6.4 & 12.4 & 75 & 69.3 & 252.4 \\
\multirow{2}{*}{ March } & 2015 & 19.0 & 7.7 & 13.3 & 75 & 72.9 & 290.1 \\
& 2016 & 22.6 & 9.4 & 16.0 & 69 & 58.8 & 251.9 \\
April & 2015 & 22.7 & 11.7 & 17.2 & 71 & 87.6 & 323.4 \\
& 2016 & 24.5 & 11.6 & 18.0 & 70 & 63.2 & 359.2 \\
May & 2015 & 25.9 & 13.7 & 19.8 & 63 & 95.7 & 606.7 \\
& 2016 & 30.0 & 18.6 & 24.3 & 62 & 87.1 & 593.8 \\
& 2015 & 30.2 & 18.8 & 24.5 & 62 & 114.6 & 715.0 \\
& 2016 & 30.4 & 22.8 & 26.6 & 58 & 97.0 & 647.0 \\
\hline
\end{tabular}

Source:Meteorological Station at Sakha Agricultural Research Station $31^{\circ} 07^{-} \mathrm{N}$ Latitude, $30^{\circ} 57^{-}$E Longitude with an elevation of about 6 metres above mean sea level (MSL). 


\section{Studied characters}

Vegetative growth characteristics

Samples consists from ten guarded plants were randomly chosen from the $2^{\text {nd }}$ row and $1^{\text {st }}$ wide furrow of each plot at 90, 105 and 120 days after transplanting (DAT) and the following data were recorded for each time: Plant height $(\mathrm{cm})$, plant fresh weight $(\mathrm{g})$, plant dry weight $(\mathrm{g})$ and leaf area per plant $\left(\mathrm{cm}^{2}\right)$,which calculated according to the following formula:

Leaf area $\left(\mathrm{cm}^{2} /\right.$ plant $)=0.000199+(1.277 \times$ leaf length $\times$ leaf diameter at a distance of $25 \%$ of the total leaf length ) (Corcoles et al., 2015).

\section{Total bulb yield and its components}

At harvest time, the experiment was harvested when $50 \%$ of tops were down. After that, the plants were cured for 15 days under the traditional field conditions then dried leaves were removed. The following yield parameters were recorded: total bulbs yield (ton/fad), marketable bulbs yield (ton/fad), culls bulb yield (ton/fad) and average weight of bulb (g).

\section{Bulb quality}

At harvest time, 10 bulbs were randomly taken as a representative sample from each experimental plot and the following physical bulb characteristics were recorded: bulb diameter $(\mathrm{cm})$, bulb length $(\mathrm{cm})$, shape index, total soluble solids (T.S.S.) and percentage of dry matter in bulbs (D.M. \%).

\section{Disease parameters}

The following diseases were determined for each experimental plot as a percentage from the plants area at the same time in both seasons.

D.1. Downy mildew disease percentage.

D.2. Purple blotch disease percentage.

\section{Economic evaluation}

E.1.Gross return from each treatment was calculated in Egyptian pounds (L.E.).

E.2. Total return $=$ Price of onion yield (L.E.).

E.3. Net return faddan ${ }^{-1}=$ total return-(fixed and variable cost of onion).

E.4.Benefit cost ratio $=$ Gross return/Cost of cultivation.

One ton of onion $=1400$ L.E., in 2014/15 and $2015 / 16$, seasons, the average prices were taken from the local market price. Economic valuation was done using the method described by CIMMYT (1988). Statistical analysis

All obtained data were statistically analyzed according to the technique of analysis of variance
(ANOVA) as published by Gomez \& Gomez (1984) by using "MSTAT-C" computer software package. Treatments means were compared according to Duncan's multiple range test (Duncan, 1955).

\section{Results and Discussions}

Data in Tables 3, 4, 5 and 6 reveal that the three transplanting dates significantly varied in their effect at all samples in both seasons. $15^{\text {th }}$ December transplanting date produced the highest values of plant height, plant fresh and dry weight and leaf area/ plant $\left(\mathrm{cm}^{2}\right)$ followed by $1^{\text {st }}$ January and $15^{\text {th }}$ January descending at 90,105 and 120 DAT during both growing seasons. These differences may be due to the suitable conditions during growth stages of plant at $15^{\text {th }}$ transplanting date that enhance growth and increase vegetative growth. Such differences were found by Al Abdulsalam \& Hamaiel (2004), Bhatt et al. (2007), Bardisi et al. (2013), Bharti \& Ram (2014), Misra et al. (2014) and Ali et al. (2016 b).

The difference between both planting methods was significant at all samples dates in the two seasons. Transplanting onions on wide furrows method significantly produced the tallest plants with the heaviest plant fresh and dry weight, as well as the highest values of leaf area/plant compared with planting in ridges at the three sampling dates in both seasons (Tables 3, 4, 5 and 6). The superiority of wide furrows system in increasing plant height may be due to help the plants to gain suitable irrigation water than the plants in ridges system consequently improving the plant growth and height. Such findings were reported by Farrag (1995), Kanwar \& Akbar (2013) and Geries et al. (2015).

Regarding plant density effect, it is clear that increasing plant density from 30 to 60 plants $/ \mathrm{m}^{2}$ significantly increased plant height during both seasons (Tables 3, 4, 5 and 6). The tallest plants were obtained from planting 60 plants $/ \mathrm{m}^{2}$, while the shortest ones were found from planting 30 plants $/ \mathrm{m}^{2}$ during the two growing seasons. The superiority of high density in increasing plant height might be due to shading under conditions of dense planting, which led to reducing light intensity and to competition for sunlight making the plants grow taller allowing them to exploit light to a maximum in denser plant populations. The highest plant fresh and dry weight and leaf area/plant were always belonged to the lowest plant density descendingly followed by 45 and 60 plants $/ \mathrm{m}^{2}$ and this trend was true in both seasons. Similar founds were recorded by Mcgeary (1985), Farrag (1995), Rizk (1997), Singh et al. (2001), Bardisi et al. (2013) and Ali et al. (2016 a). 
TABLE 3. Effect of planting date, method and density on plant height $(\mathrm{cm})$ of onion as well as their interactions at different sampling dates in 2014/15 and 2015/16 seasons.

\begin{tabular}{|c|c|c|c|c|c|c|}
\hline \multirow{4}{*}{ Treatment } & \multicolumn{6}{|c|}{ Plant height (cm) } \\
\hline & \multicolumn{3}{|c|}{$2014 / 15$} & \multicolumn{3}{|c|}{$2015 / 16$} \\
\hline & \multicolumn{6}{|c|}{ Days after transplanting (DAT) } \\
\hline & 90 & 105 & 120 & 90 & 105 & 120 \\
\hline Planting date $(\mathrm{A})$ : & & & & & & \\
\hline $15^{\text {th }}$ December & $60.27 \mathrm{a}$ & $71.69 \mathrm{a}$ & $74.37 \mathrm{a}$ & $61.74 \mathrm{a}$ & $72.22 \mathrm{a}$ & $79.82 \mathrm{a}$ \\
\hline $1^{\text {st }}$ January & $55.50 \mathrm{~b}$ & $64.13 \mathrm{~b}$ & $62.44 \mathrm{~b}$ & $57.55 \mathrm{ab}$ & $70.38 \mathrm{~b}$ & $68.42 \mathrm{~b}$ \\
\hline $15^{\text {th }}$ January & $52.76 \mathrm{c}$ & $58.56 \mathrm{c}$ & $53.54 \mathrm{c}$ & $53.19 \mathrm{~b}$ & $68.47 \mathrm{c}$ & $55.90 \mathrm{c}$ \\
\hline F-test & $* *$ & $* *$ & $* *$ & * & $* *$ & $* *$ \\
\hline $\begin{array}{l}\text { Planting method (B): } \\
\text { Ridges }\end{array}$ & $54.75 \mathrm{~b}$ & $62.77 \mathrm{~b}$ & $62.46 \mathrm{~b}$ & $54.38 \mathrm{~b}$ & 69.58 & $65.95 \mathrm{~b}$ \\
\hline Wide furrows & $57.59 \mathrm{a}$ & $66.82 \mathrm{a}$ & $64.44 \mathrm{a}$ & $60.61 \mathrm{a}$ & 71.13 & $70.14 \mathrm{a}$ \\
\hline $\begin{array}{l}\text { F-test } \\
\text { Planting density }(\mathrm{C}) \text { : }\end{array}$ & * & * & $* *$ & $* *$ & N.S & $* *$ \\
\hline 30 plants $/ \mathrm{m}^{2}$ & $50.95 \mathrm{c}$ & $62.63 \mathrm{~b}$ & $61.30 \mathrm{c}$ & $52.94 \mathrm{~b}$ & $67.05 \mathrm{~b}$ & $65.73 \mathrm{~b}$ \\
\hline 45 plants $/ \mathrm{m}^{2}$ & $56.30 \mathrm{~b}$ & $64.67 \mathrm{ab}$ & $63.17 \mathrm{~b}$ & $58.83 \mathrm{a}$ & 70.98 a & $67.60 \mathrm{ab}$ \\
\hline 60 plants $/ \mathrm{m}^{2}$ & $61.27 \mathrm{a}$ & $67.08 \mathrm{a}$ & $65.89 \mathrm{a}$ & $60.71 \mathrm{a}$ & $73.03 \mathrm{a}$ & $70.81 \mathrm{a}$ \\
\hline $\begin{array}{l}\text { F-test } \\
\text { Interaction : }\end{array}$ & $* *$ & $*$ & $* *$ & $* *$ & $* *$ & $* *$ \\
\hline A X B & N.S & N.S & N.S & N.S & N.S & $*$ \\
\hline $\mathrm{AXC}$ & N.S & N.S & N.S & N.S & N.S & N.S \\
\hline B X C & N.S & N.S & $*$ & N.S & N.S & N.S \\
\hline A X B X C & N.S & N.S & N.S & N.S & N.S & N.S \\
\hline
\end{tabular}

*, ** and N.S.: significant, highly and not significant, respectively.Means within the same column for each factor followed by the same letter are not significantly different, according to DMRT at 0.05 level.

TABLE 4. Plant fresh weight (g) of onion as influenced by planting date, method and density and their interactions at different sampling dates in 2014/15 and 2015/16 seasons.

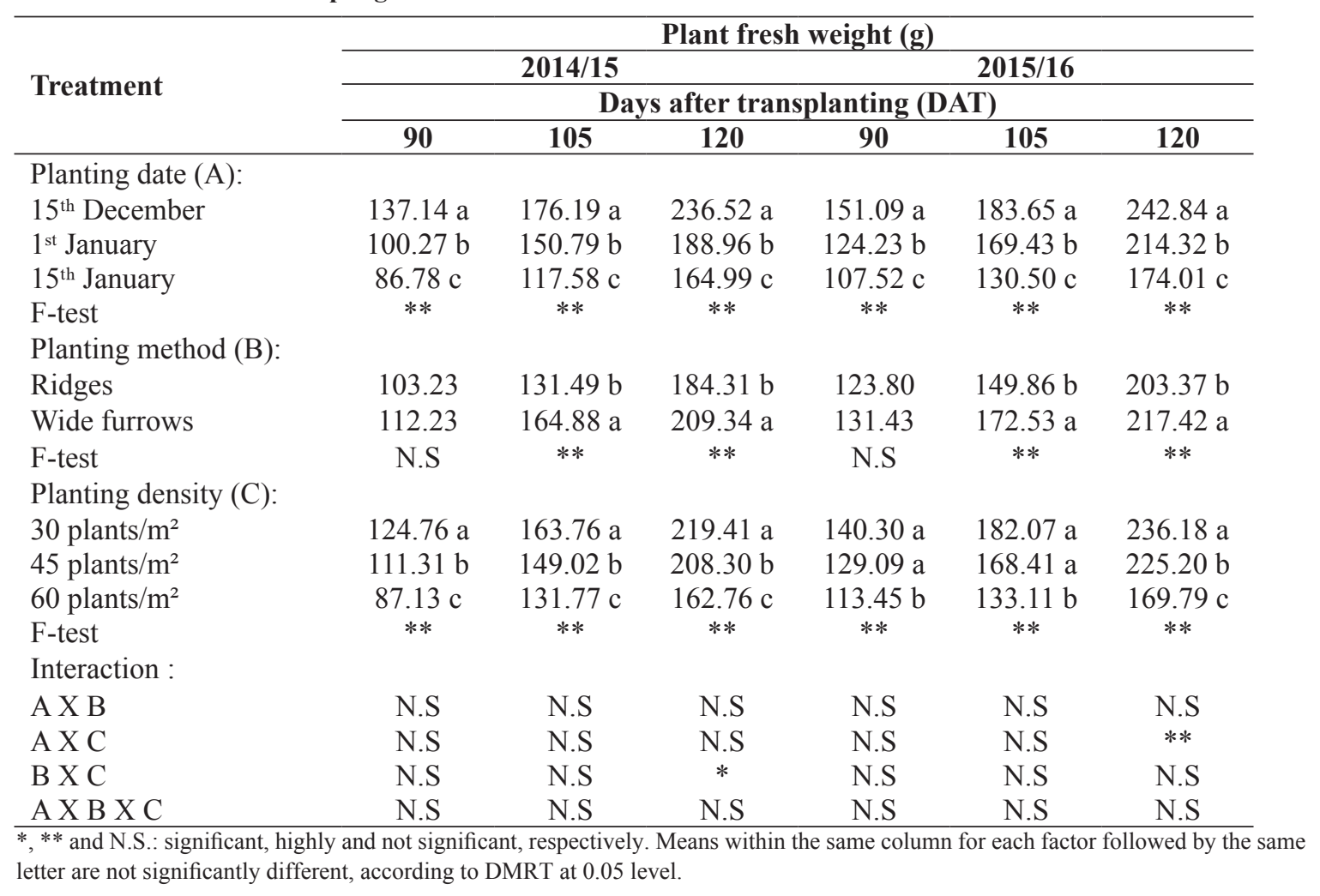


TABLE 5. Effect of planting date, method and density and their interactions on plant dry weight (g) of onion at 90,105 and 120 DAT in 2014/15 and 2015/16 seasons.

\begin{tabular}{|c|c|c|c|c|c|c|}
\hline \multirow{4}{*}{ Treatment } & \multicolumn{6}{|c|}{ Plant dry weight (g) } \\
\hline & \multicolumn{3}{|c|}{$2014 / 15$} & \multicolumn{3}{|c|}{$2015 / 16$} \\
\hline & \multicolumn{6}{|c|}{ Days after transplanting (DAT) } \\
\hline & 90 & 105 & 120 & 90 & 105 & 120 \\
\hline \multicolumn{7}{|l|}{ Planting date (A): } \\
\hline $15^{\text {th }}$ December & $9.42 \mathrm{a}$ & $16.71 \mathrm{a}$ & $22.21 \mathrm{a}$ & $10.61 \mathrm{a}$ & $17.43 \mathrm{a}$ & $23.16 \mathrm{a}$ \\
\hline $1^{\text {st }}$ January & $7.35 \mathrm{~b}$ & $12.42 \mathrm{~b}$ & $16.17 \mathrm{~b}$ & $8.13 \mathrm{~b}$ & $14.79 \mathrm{~b}$ & $19.86 \mathrm{~b}$ \\
\hline $15^{\text {th }}$ January & $5.78 \mathrm{c}$ & $8.81 \mathrm{c}$ & $12.82 \mathrm{c}$ & $5.80 \mathrm{c}$ & $10.25 \mathrm{c}$ & $14.71 \mathrm{c}$ \\
\hline F-test & $* *$ & $* *$ & $* *$ & $* *$ & $* *$ & $* *$ \\
\hline \multicolumn{7}{|l|}{ Planting method (B): } \\
\hline Ridges & $7.02 \mathrm{~b}$ & $11.48 \mathrm{~b}$ & $16.10 \mathrm{~b}$ & $7.52 \mathrm{~b}$ & $13.47 \mathrm{~b}$ & $17.61 \mathrm{~b}$ \\
\hline Wide furrows & $8.00 \mathrm{a}$ & $13.81 \mathrm{a}$ & $18.04 \mathrm{a}$ & $8.84 \mathrm{a}$ & $14.84 \mathrm{a}$ & $20.88 \mathrm{a}$ \\
\hline F-test & $*$ & $* *$ & $* *$ & $* *$ & $* *$ & $* *$ \\
\hline \multicolumn{7}{|l|}{ Planting density $(\mathrm{C})$ : } \\
\hline 30 plants $/ \mathrm{m}^{2}$ & $8.75 \mathrm{a}$ & $14.30 \mathrm{a}$ & $19.61 \mathrm{a}$ & $9.83 \mathrm{a}$ & $16.64 \mathrm{a}$ & $21.80 \mathrm{a}$ \\
\hline 45 plants $/ \mathrm{m}^{2}$ & $7.77 \mathrm{~b}$ & $12.79 \mathrm{~b}$ & $16.55 \mathrm{~b}$ & $8.45 \mathrm{~b}$ & $14.38 \mathrm{~b}$ & $19.45 \mathrm{~b}$ \\
\hline 60 plants $/ \mathrm{m}^{2}$ & $6.01 \mathrm{c}$ & $10.84 \mathrm{c}$ & $14.65 \mathrm{c}$ & $6.27 \mathrm{c}$ & $11.46 \mathrm{c}$ & $16.48 \mathrm{c}$ \\
\hline F-test & $* *$ & $* *$ & $* *$ & $* *$ & $* *$ & $* *$ \\
\hline \multicolumn{7}{|l|}{ Interaction : } \\
\hline $\mathrm{A} X \mathrm{~B}$ & N.S & N.S & N.S & N.S & N.S & N.S \\
\hline $\mathrm{AXC}$ & N.S & N.S & $*$ & $* *$ & N.S & N.S \\
\hline B X C & N.S & N.S & N.S & N.S & N.S & N.S \\
\hline $\mathrm{A} \times \mathrm{B} \times \mathrm{C}$ & N.S & N.S & N.S & N.S & N.S & N.S \\
\hline
\end{tabular}

TABLE 6. Leaf area/plant $\left(\mathrm{cm}^{2}\right)$ of onion Giza Red as affected by planting date, method and density and their interactions at different sampling dates in 2014/15 and 2015/16 seasons.

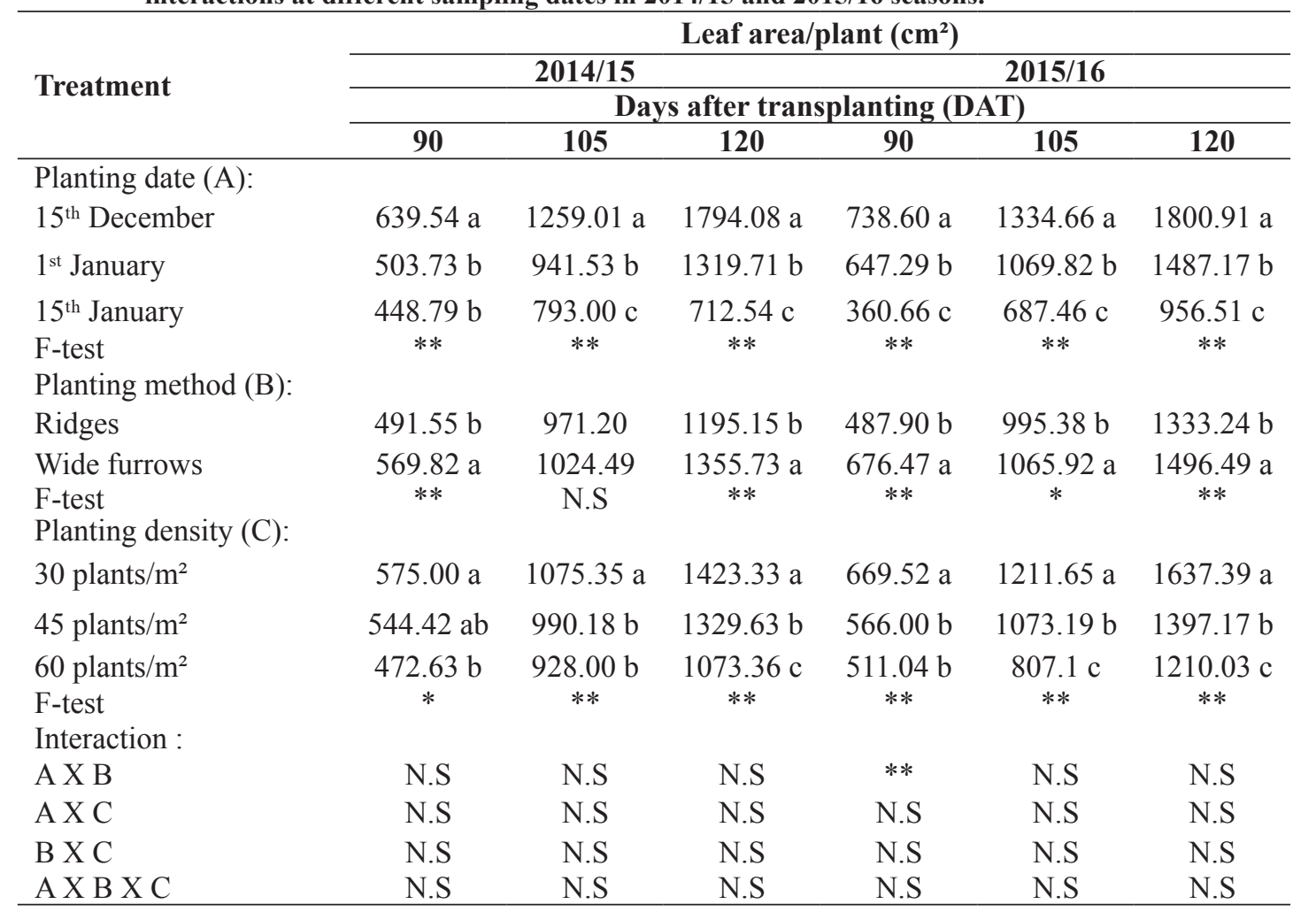

\footnotetext{
*,** and N.S.: significant, highly and not significant, respectively. Means within the same column for each factor followed by the same
} letter are not significantly different, according to DMRT at 0.05 level. 
Concerning the interaction among the experimental factors, Table 7 shows that transplanting onions at $15^{\text {th }}$ December in wide furrows planting system significantly recorded the tallest plants and leaf area/plant. Planting 30 plants $/ \mathrm{m}^{2}$ in wide furrows recorded the highest values of plant fresh weight, while the tallest plant height were produced by planting onions in wide furrows with 60 plants $/ \mathrm{m}^{2}$. On the other hand, the highest plant fresh and dry weight was produced when Giza Red onion cultivar transplanted at $15^{\text {th }}$ December with 30 plants $/ \mathrm{m}^{2}$, while the lowest one was obtained by transplanting at $15^{\text {th }}$ January with 60 plants $/ \mathrm{m}^{2}$.

TABLE 7. Highest values of plant height, plant fresh and dry weight and leaf area/plant of Giza Red onion as affected by significant interaction among the experimental factors in 2014/15 and 2015/16 seasons.

\begin{tabular}{lccc}
\hline Variable & \multicolumn{1}{c}{ Season } & $\begin{array}{c}\text { Highest } \\
\text { values }\end{array}$ & Treatment \\
\hline Plant height $(\mathrm{cm})$ & 120DAT (2014/15) & 66.49 & $\begin{array}{c}\text { Wide furrowsx60 plants } / \mathrm{m}^{2} \\
15^{\text {th }} \text { Decemberx Wide furrows }\end{array}$ \\
& 120DAT (2015/16) & 82.06 & Wide furrows x 30 plants $/ \mathrm{m}^{2}$ \\
Plant fresh weight $(\mathrm{g})$ & 120DAT $(2014 / 15)$ & 239.32 & $15^{\text {th }}$ Decemberx 30 plants $/ \mathrm{m}^{2}$ \\
& 120DAT(2015/16) & 274.94 & \\
Plant dry weight $(\mathrm{g})$ & 120 DAT(2014/15) & 26.71 & $15^{\text {th }}$ December x 30 plants $/ \mathrm{m}^{2}$ \\
& 90 DAT(2015/16) & 13.27 & \\
Leaf area/plant $\left(\mathrm{cm}^{2}\right)$ & 90 DAT(2015/16) & 894.80 & $15^{\text {th }}$ Decemberx Wide furrows \\
\hline
\end{tabular}

\section{Total bulb yield and its components}

With respect to onion transplanting date impact, the three tested dates showed a significant difference in total bulbs yield of onion in the $1^{\text {st }}$ and the $2^{\text {nd }}$ seasons (Table 8 ). The highest total yield of bulbs, marketable bulbs yield and average bulb weight were produced by the early transplanting date $\left(15^{\text {th }}\right.$ December) in both growing seasons. On the contrary, the heaviest culls bulb yield/fad was obtained by transplanting onions at $15^{\text {th }}$ January at 2014/15 and 2015/16 seasons. These results are might be due to high average temperature in early transplanting date and low average temperature in late transplanting date during the growth period, which contributed to good foliage growth and formation of ample canopy able to enhance photosynthesis, hence increasing dry matter accumulation, bulb weight as well as total bulbs yield/fad. Results of Mosleh Ud - Deen (2008), George et al. (2009), Ibrahim \& Adesiyun (2009), Caruso et al. (2013), Kandil et al. (2013), Mostafizur (2013), Bharti \& Ram (2014), Misra et al. (2014) and Ali et al. (2016 b) are in agreement with our conclusions.

As for the effect of planting method, it is clear from Tables 8 that there were significant differences between planting in wide furrows and ridges in both growing seasons. The data reveal that, the maximum total bulb yield and its components was always associated to planting in wide furrows comparing with planting in ridges in 2014/15 and 2015/16 seasons. Ahmed \& Hassan (1978), Farrag (1995), Nawer (2006), Kanwar \& Akbar (2013) and Geries et al. (2015) came to the same results.

Data of both seasons located in Table 8 indicate that total yield of bulbs significantly affected by plant density during this study. Total and marketable bulbs yield were significantly highest at 45 plants $/ \mathrm{m}^{2}$ followed by 60 plants/ $\mathrm{m}^{2}$ and the reverse was true for 30 plants $/ \mathrm{m}^{2}$.As for the maximum culls bulb yield per faddan was obtained by planting at 60 plants $/ \mathrm{m}^{2}$. The increasing in total bulbs yield/fad by raising plant density might be attributed mainly to the increase in number of plants/fad, which was great enough to compensate the detected decrease in bulb weight. So, the highest average bulb weight was always belonged to the lowest density, while the lightest average bulb weight was obtained from the highest density, without significant difference between 30 and 45 plants $/ \mathrm{m}^{2}$ in 2015/16 season only, These results are in agreement with those mentioned by Awas et al. (2010), Nguthi (2013), Saud et al. (2013), Misra et al. (2014), Geries et al. (2015), Islam et al. (2015), Siliquini et al. (2015), Shock et al. (2015), Ali et al. (2016 a \& b) and Haq et al. (2016). 
TABLE 8. Bulb yield (ton/fad) and its components as influenced by planting date, method and density of Giza Red onion and their interactions in 2014/15 and 2014/15 seasons.

\begin{tabular}{|c|c|c|c|c|}
\hline \multirow[b]{2}{*}{ Treatment } & \multicolumn{4}{|c|}{$2014 / 15$} \\
\hline & $\begin{array}{c}\text { Total } \\
\text { bulbs yield } \\
\text { (ton/fad) }\end{array}$ & $\begin{array}{c}\text { Marketable } \\
\text { bulbs yield } \\
\text { (ton/fad) }\end{array}$ & $\begin{array}{c}\text { Culls yield } \\
\text { (ton/fad) }\end{array}$ & $\begin{array}{c}\text { Average bulb } \\
\text { weight (g) }\end{array}$ \\
\hline \multicolumn{5}{|l|}{ Planting date (A): } \\
\hline $15^{\text {th }}$ December & $12.82 \mathrm{a}$ & $11.07 \mathrm{a}$ & $1.74 \mathrm{~b}$ & $104.29 \mathrm{a}$ \\
\hline $1^{\text {st }}$ January & $11.64 \mathrm{~b}$ & $10.06 \mathrm{~b}$ & $1.58 \mathrm{~b}$ & $84.96 \mathrm{~b}$ \\
\hline $15^{\text {th }}$ January & $10.11 \mathrm{c}$ & $8.15 \mathrm{c}$ & $1.96 \mathrm{a}$ & $67.05 \mathrm{c}$ \\
\hline F-test & $* *$ & $* *$ & $* *$ & $* *$ \\
\hline $\begin{array}{l}\text { Planting method (B): } \\
\text { Ridges }\end{array}$ & $11.03 \mathrm{~b}$ & $9.37 \mathrm{~b}$ & $1.66 \mathrm{~b}$ & $79.09 \mathrm{~b}$ \\
\hline Wide furrows & $12.01 \mathrm{a}$ & $10.15 \mathrm{a}$ & $1.86 \mathrm{a}$ & $91.78 \mathrm{a}$ \\
\hline F-test & $* *$ & $* *$ & $* *$ & $* *$ \\
\hline \multicolumn{5}{|l|}{ Planting density (C): } \\
\hline 30 plants $/ \mathrm{m}^{2}$ & $10.16 \mathrm{c}$ & $8.80 \mathrm{c}$ & $1.36 \mathrm{c}$ & $97.66 \mathrm{a}$ \\
\hline 45 plants $/ \mathrm{m}^{2}$ & $12.49 \mathrm{a}$ & $10.70 \mathrm{a}$ & $1.79 \mathrm{~b}$ & $85.31 \mathrm{~b}$ \\
\hline 60 plants $/ \mathrm{m}^{2}$ & $11.91 \mathrm{~b}$ & $9.78 \mathrm{~b}$ & $2.14 \mathrm{a}$ & $73.32 \mathrm{c}$ \\
\hline F-test & $* *$ & $* *$ & $* *$ & $* *$ \\
\hline $\begin{array}{l}\text { Interaction : } \\
\text { A X B }\end{array}$ & * & $*$ & N.S & $* *$ \\
\hline $\mathrm{A} \times \mathrm{C}$ & N.S & $*$ & $*$ & $* *$ \\
\hline $\mathrm{B} \times \mathrm{C}$ & $* *$ & N.S & $* *$ & N.S \\
\hline \multirow[t]{2}{*}{$\mathrm{A} \times \mathrm{B} \times \mathrm{C}$} & N.S & N.S & $* *$ & $* *$ \\
\hline & \multicolumn{4}{|c|}{$2015 / 16$} \\
\hline Planting date (A): & & & & \\
\hline $15^{\text {th }}$ December & $14.28 \mathrm{a}$ & $12.75 \mathrm{a}$ & $1.54 \mathrm{~b}$ & $106.51 \mathrm{a}$ \\
\hline $1^{\text {st }}$ January & $12.50 \mathrm{~b}$ & $10.97 \mathrm{~b}$ & $1.53 \mathrm{~b}$ & $93.91 \mathrm{~b}$ \\
\hline $15^{\text {th }}$ January & $11.22 \mathrm{c}$ & $9.28 \mathrm{c}$ & $1.94 \mathrm{a}$ & $75.99 \mathrm{c}$ \\
\hline F-test & $* *$ & $* *$ & $* *$ & $* *$ \\
\hline $\begin{array}{l}\text { Planting method (B): } \\
\text { Ridges } \\
\text { Wide furrows }\end{array}$ & $\begin{array}{l}11.66 \mathrm{~b} \\
13.68 \mathrm{a}\end{array}$ & $\begin{array}{l}10.17 \mathrm{~b} \\
11.83 \mathrm{a}\end{array}$ & $\begin{array}{l}1.50 \mathrm{~b} \\
1.85 \mathrm{a}\end{array}$ & $\begin{array}{l}85.42 \mathrm{~b} \\
98.85 \mathrm{a}\end{array}$ \\
\hline F-test & $* *$ & $* *$ & $* *$ & $* *$ \\
\hline $\begin{array}{l}\text { Planting density }(\mathrm{C}) \text { : } \\
30 \text { plants } / \mathrm{m}^{2} \\
45 \text { plants } / \mathrm{m}^{2} \\
60 \text { plants } / \mathrm{m}^{2}\end{array}$ & $\begin{array}{l}10.66 \mathrm{c} \\
14.17 \mathrm{a} \\
13.18 \mathrm{~b}\end{array}$ & $\begin{array}{l}9.49 \mathrm{c} \\
12.41 \mathrm{a} \\
11.10 \mathrm{~b}\end{array}$ & $\begin{array}{l}1.17 \mathrm{c} \\
1.76 \mathrm{~b} \\
2.08 \mathrm{a}\end{array}$ & $\begin{array}{l}101.92 \mathrm{a} \\
94.44 \mathrm{a} \\
80.04 \mathrm{~b}\end{array}$ \\
\hline F-test & $* *$ & $* *$ & $* *$ & $* *$ \\
\hline $\begin{array}{l}\text { Interaction : } \\
\mathrm{A} \times \mathrm{B}\end{array}$ & $* *$ & $*$ & $*$ & $*$ \\
\hline $\mathrm{AXC}$ & $* *$ & $*$ & N.S & N.S \\
\hline B X C & N.S & N.S & N.S & N.S \\
\hline $\mathrm{A} \times \mathrm{B} \times \mathrm{C}$ & $*$ & N.S & N.S & N.S \\
\hline
\end{tabular}

*,** and N.S.: significant, highly and not significant, respectively. Means within the same column for each factor followed by the same letter are not significantly different, according to DMRT at 0.05 level. 
As for the interaction among the experimental factors, Table 9 shows clearly that transplanting onion at $15^{\text {th }}$ December date under wide furrows planting method significantly produced the highest total bulbs yield, marketable bulbs yield, culls yield/fad and average bulb weight. Planting 45 plants $/ \mathrm{m}^{2}$ of onion at $15^{\text {th }}$ December recorded the highest values of total bulbs yield and marketable bulbs yield. While, the highest average bulb weight was belonged to $15^{\text {th }}$ January date, under the density of 30 plants $/ \mathrm{m}^{2}$. The maximum total bulb yield was obtained by transplanting onions at $15^{\text {th }}$ December in wide furrows planting method with the medium plant density (45 plants $/ \mathrm{m}^{2}$ ).

TABLE 9. Highest values of total bulbs yield, marketable bulbs yield, culls yield/fad and average bulb weight of Giza Red onion as affected by significant interaction among the experimental factors in 2014/15 and 2015/16 seasons.

\begin{tabular}{|c|c|c|c|}
\hline Variable & Season & Highest values & Treatment \\
\hline \multirow{6}{*}{$\begin{array}{l}\text { Total bulbs yield } \\
\quad \text { (ton/fad) }\end{array}$} & $2014 / 15$ & 13.62 & \multirow{3}{*}{$15^{\text {th }}$ December $x$ Wide furrows } \\
\hline & & & \\
\hline & $2015 / 16$ & 15.58 & \\
\hline & $2015 / 16$ & 15.30 & $15^{\text {th }}$ December x 45 plants $/ \mathrm{m}^{2}$ \\
\hline & $2014 / 15$ & 13.36 & Wide furrows x 45 plants $/ \mathrm{m}^{2}$ \\
\hline & $2015 / 16$ & 16.51 & $15^{\text {th }}$ December $x$ Wide furrows $x 45$ plants $/ \mathrm{m}^{2}$ \\
\hline \multirow{4}{*}{$\begin{array}{l}\text { Marketable bulbs } \\
\text { yield (ton/fad) }\end{array}$} & $2014 / 15$ & 11.74 & \multirow{2}{*}{$15^{\text {th }}$ December $x$ Wide furrows } \\
\hline & $2015 / 16$ & 13.87 & \\
\hline & $2014 / 15$ & 12.08 & \multirow{2}{*}{$15^{\text {th }}$ December $x 45$ plants $/ \mathrm{m}^{2}$} \\
\hline & $2015 / 16$ & 13.65 & \\
\hline \multirow{4}{*}{$\begin{array}{l}\text { Culls yield } \\
\text { (ton/fad) }\end{array}$} & $2015 / 16$ & 2.09 & $15^{\text {th }}$ January $x$ Wide furrows \\
\hline & $2014 / 15$ & 2.30 & $15^{\text {th }}$ January x 60 plants $/ \mathrm{m}^{2}$ \\
\hline & $2014 / 15$ & 2.19 & Ridges x 60 plants $/ \mathrm{m}^{2}$ \\
\hline & $2014 / 15$ & 2.50 & $15^{\text {th }}$ January $x$ Ridgesx 60 plants $/ \mathrm{m}^{2}$ \\
\hline \multirow{4}{*}{$\begin{array}{l}\text { Average bulb } \\
\text { weight } \\
\text { (g) }\end{array}$} & $2014 / 15$ & 113.50 & \multirow{2}{*}{$15^{\text {th }}$ December $x$ Wide furrows } \\
\hline & $2015 / 16$ & 116.48 & \\
\hline & $2014 / 15$ & 124.38 & $15^{\text {th }}$ December $x 30$ plants $/ \mathrm{m}^{2}$ \\
\hline & $2014 / 15$ & 130.93 & $15^{\text {th }}$ December $x$ Wide furrows $x 30$ plants $/ \mathrm{m}^{2}$ \\
\hline \multicolumn{3}{|c|}{$\begin{array}{l}\text { Bulb quality } \\
\text { There was a significant effect for onion } \\
\text { transplanting date on the bulb diameter in both } \\
\text { seasons (Table 10). Transplanting onions at } \\
15^{\text {th }} \text { December produced the highest value of bulb } \\
\text { diameter ( } 7.32 \text { and } 7.54 \mathrm{~cm}) \text {, shape index,TSS \% }(14.88 \\
\text { and } 15.22 \% \text { ) and D.M. (17.07 and } 18.10 \%) \text { while } \\
1^{\text {st }} \text { January date gave the second heights values, } \\
\text { and the last transplanting date (15 January) } \\
\text { gave the lowest one in the two growing seasons, } \\
\text { respectively. These results are in compatible with } \\
\text { those stated by Leilah et al. (2003), Caruso et al. } \\
\text { (2013), Bharti \& Ram (2014), Misra et al. (2014) } \\
\text { and Ali et al. ( } 2016 \mathrm{~b}) \text {. }\end{array}$} & $\begin{array}{l}\text { It is clear from the data in Table } 10 \text { that } \\
\text { planting method had a significant impact on bulb } \\
\text { quality in both growing seasons. The maximum } \\
\text { values of bulb diameter, T.S.S \% and D.M. \% } \\
\text { were obtained by planting in wide furrows and } \\
\text { the reverse was true for planting in ridges during } \\
\text { the both growing seasons. The decrease in T.S.S } \\
\text { due to delaying transplanting date from } 15^{\text {th }} \\
\text { December to } 1^{\text {st }} \text { January agreed with (Coolong } \\
\& \text { Randle, } 2003 \text { ), who found out that the soluble } \\
\text { solid content of mature bulbs had a negative linear } \\
\text { response to increasing temperature. These results } \\
\text { are in general agreement with those obtained by } \\
\text { Kandil et al. (2013), Kanwar \& Akbar (2013) and } \\
\text { Bharti \& Ram (2014). }\end{array}$ \\
\hline
\end{tabular}


TABLE 10. Bulb quality as influenced by planting date, method and density and their interactions in 2014/15 and 2015/16 seasons.

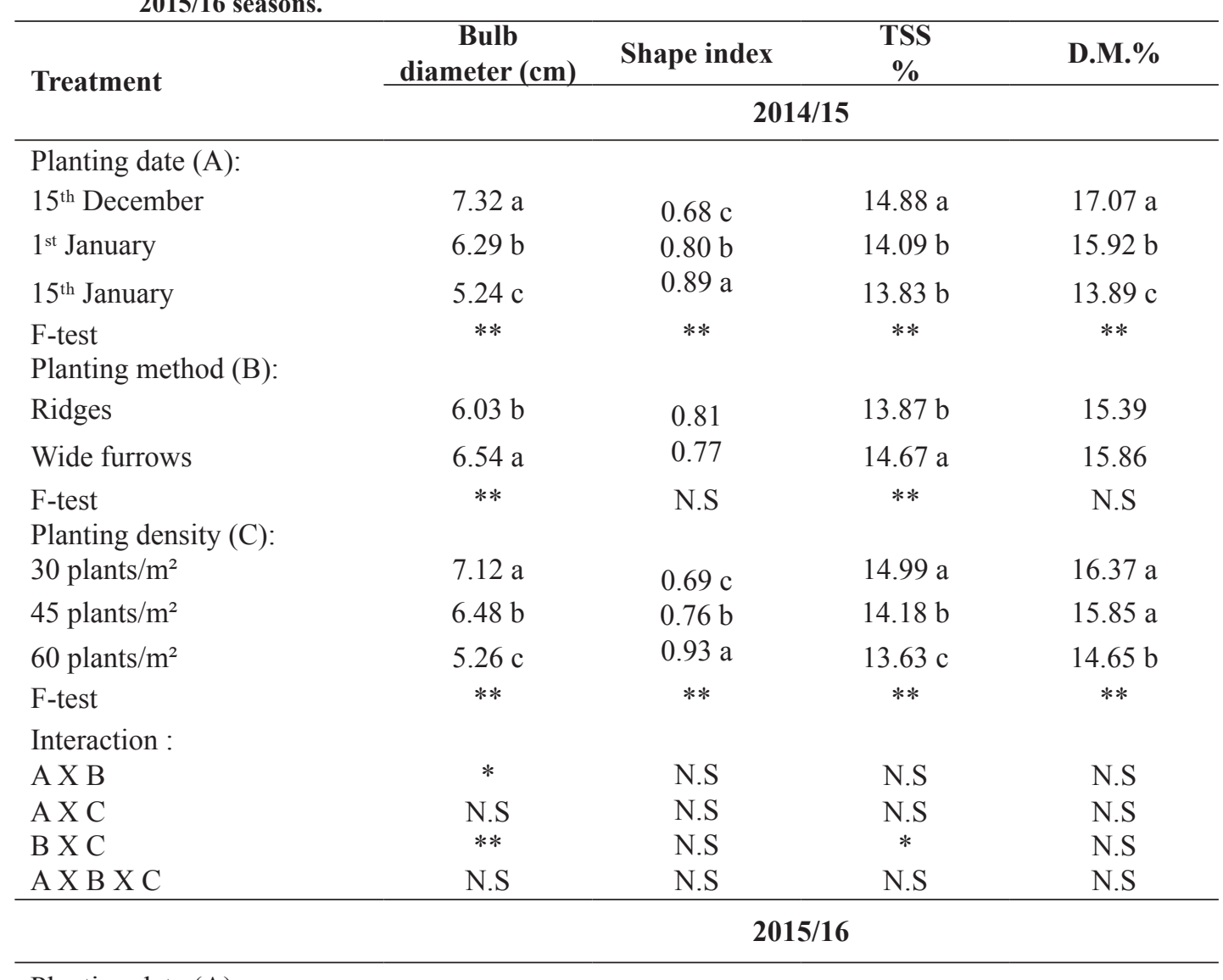

\begin{tabular}{|c|c|c|c|c|}
\hline \multicolumn{5}{|c|}{ Planting date (A): } \\
\hline $15^{\text {th }}$ December & $7.54 \mathrm{a}$ & \multirow{3}{*}{$\begin{array}{c}0.72 \mathrm{~b} \\
0.80 \mathrm{ab} \\
0.86 \mathrm{a}\end{array}$} & $15.22 \mathrm{a}$ & $18.10 \mathrm{a}$ \\
\hline $1^{\text {st }}$ January & $6.36 \mathrm{~b}$ & & $14.57 \mathrm{~b}$ & $16.17 \mathrm{~b}$ \\
\hline $15^{\text {th }}$ January & $5.74 \mathrm{c}$ & & $14.18 \mathrm{~b}$ & $14.62 \mathrm{c}$ \\
\hline F-test & $* *$ & $*$ & $*$ & $* *$ \\
\hline \multicolumn{5}{|c|}{ Planting method (B): } \\
\hline Ridges & $6.36 \mathrm{~b}$ & \multirow{2}{*}{$\begin{array}{l}0.82 \\
0.77\end{array}$} & $14.31 \mathrm{~b}$ & $15.97 \mathrm{~b}$ \\
\hline Wide furrows & $6.73 \mathrm{a}$ & & $15.00 \mathrm{a}$ & $16.61 \mathrm{a}$ \\
\hline F-test & $* *$ & N.S & $*$ & $*$ \\
\hline \multicolumn{5}{|c|}{ Planting density $(\mathrm{C})$ : } \\
\hline 30 plants $/ \mathrm{m}^{2}$ & $7.12 \mathrm{a}$ & \multirow{3}{*}{$\begin{array}{l}0.72 \mathrm{c} \\
0.78 \mathrm{~b} \\
0.88 \mathrm{a}\end{array}$} & $15.16 \mathrm{a}$ & $17.08 \mathrm{a}$ \\
\hline 45 plants $/ \mathrm{m}^{2}$ & $6.64 \mathrm{~b}$ & & $14.83 \mathrm{a}$ & $16.20 \mathrm{~b}$ \\
\hline 60 plants $/ \mathrm{m}^{2}$ & $5.88 \mathrm{c}$ & & $13.98 \mathrm{~b}$ & $15.61 \mathrm{c}$ \\
\hline F-test & $* *$ & $* *$ & $* *$ & $* *$ \\
\hline \multicolumn{5}{|l|}{ Interaction : } \\
\hline A X B & $* *$ & N.S & N.S & N.S \\
\hline $\mathrm{AXC}$ & N.S & N.S & N.S & $*$ \\
\hline B X C & N.S & N.S & N.S & N.S \\
\hline $\mathrm{A} X \mathrm{~B} \times \mathrm{C}$ & $*$ & N.S & N.S & N.S \\
\hline
\end{tabular}

*,** and N.S.: significant, highly and not significant, respectively. Means within the same column for each factor followed by the same letter are not significantly different, according to DMRT at 0.05 level. 
The bulb diameter of onion, T.S.S\% and D.M. $\%$ for the three tested plant density was arranged as follows: 30 plants $/ \mathrm{m}^{2}>45$ plants $/ \mathrm{m}^{2}>60$ plants/ $\mathrm{m}^{2}$ in both seasons of study (Table10). But, shape index values under the different plant densities were arranged as follows: 60 plants/ $\mathrm{m}^{2}>45$ plants $/ \mathrm{m}^{2}>30$ plants $/ \mathrm{m}^{2}$ in $2014 / 15$ and $2015 / 16$ growing seasons. The superiority of low density in bulb diameter may be attributed to decreased competition for nutrients and moisture at low dense plants that resulted in bulbs with larger diameter and higher bulb weight. In this connection Awas et al. (2010), Saud et al. (2013), Bosekeng \& Coetzer (2015), Geries et al. (2015), Hatridge-Eshand \& Bennett (1980), Islam et al.
(2015) and Shock et al. (2015) found similar results in onion plants.

As condition to the interaction of the experimental factors (Table 11), transplanting onion at $15^{\text {th }}$ December date under wide furrows planting system significantly produced the largest bulb diameter of onions, while the highest dry matter percentage of onion was produced from transplanting onions at $15^{\text {th }}$ December date with the lowest density (30 plants $\left./ \mathrm{m}^{2}\right)$. On the other hand, the highest values of bulb diameter and TSS \% were obtained,when planted under wide furrows planting system with the lowest plant density (30 plants $\left./ \mathrm{m}^{2}\right)$.

TABLE 11. Highest values of bulb diameter, T.S.S \%, and D.M. \% of Giza Red onion as affected by significant interaction among the experimental factors in $2014 / 2015$ and $2015 / 2016$ seasons.

$\begin{array}{lll}\text { Variable Season } \quad \text { Highest values } & \text { Treatment }\end{array}$

\begin{tabular}{|c|c|c|c|}
\hline \multirow{5}{*}{$\begin{array}{l}\text { Bulb } \\
\text { diameter } \\
(\mathrm{cm})\end{array}$} & $2014 / 15$ & 7.48 & \multirow{3}{*}{$15^{\text {th }}$ December $x$ Wide furrows } \\
\hline & & & \\
\hline & $2015 / 16$ & 7.49 & \\
\hline & $2014 / 15$ & 7.59 & Wide furrows x 30 plants $/ \mathrm{m}^{2}$ \\
\hline & $2015 / 16$ & 8.18 & $15^{\text {th }}$ December $x$ Wide furrows $x 30$ plants $/ \mathrm{m}^{2}$ \\
\hline TSS \% & $2014 / 15$ & 15.57 & Wide furrows x 30 plants $/ \mathrm{m}^{2}$ \\
\hline D.M. \% & $2014 / 15$ & 18.68 & $15^{\text {th }}$ December x 30 plants $/ \mathrm{m}^{2}$ \\
\hline
\end{tabular}

\section{Disease parameters}

The analysis of variance indicated that transplanting date had a significant effect on downy mildew and purple blotch disease percentage in onion during both seasons. The obtained data show that $15^{\text {th }}$ December transplanting date gave the highest values of downy mildew and purple blotch percentage in the first and second seasons with significant difference compared to the rest of the treatments as presented in Table 12. $1^{\text {st }}$ January transplanting date gave the second highest values, while $15^{\text {th }}$ January gave the lowest values in both seasons of study. These results may be due to the suitable weather conditions at the early transplanting date $\left(15^{\text {th }}\right.$ December $)$, which enhancing branching ability and number of green leaves per plant leading to increasing moisture percentage between plants consequently increasing downy mildew and purple blotch disease percentage.Similar findings were recorded by Maude (1990) and Schwartz \& Mohan (2008).

As for the effect of planting method system on downy mildew and purple blotch disease percentage, it is clear from Table 12 that there were significant differences between planting on wide furrows and planting in ridges in both growing seasons. The highest percentage was always belonged to planting on ridges. These results were mainly due to the increased ability of ridge planting method to hold water after irrigation than wide furrows method. 
TABLE 12. Disease parameters percentage of Giza Red onion as influenced by planting date, method and density and their interactions in 2014/15 and 2015/16 seasons.

\begin{tabular}{|c|c|c|c|c|}
\hline \multirow[b]{2}{*}{ Treatment } & \multicolumn{2}{|c|}{$2014 / 15$} & \multicolumn{2}{|c|}{ 2015/16 } \\
\hline & $\begin{array}{c}\text { Downy mildew } \\
\%\end{array}$ & $\begin{array}{c}\text { Purple blotch } \\
\%\end{array}$ & $\begin{array}{c}\text { Downy mildew } \\
\%\end{array}$ & $\begin{array}{c}\text { Purple blotch } \\
\%\end{array}$ \\
\hline \multicolumn{5}{|c|}{ Planting date (A): } \\
\hline $15^{\text {th }}$ December & $5.79 \mathrm{a}$ & $6.83 \mathrm{a}$ & $4.42 \mathrm{a}$ & $5.08 \mathrm{a}$ \\
\hline $1^{\text {st January }}$ & $4.06 \mathrm{~b}$ & $5.08 \mathrm{~b}$ & $3.73 \mathrm{a}$ & $3.78 \mathrm{~b}$ \\
\hline $15^{\text {th }}$ January & $3.27 \mathrm{c}$ & $3.96 \mathrm{c}$ & $2.72 \mathrm{~b}$ & $2.31 \mathrm{c}$ \\
\hline F-test & $* *$ & $* *$ & $* *$ & $* *$ \\
\hline \multicolumn{5}{|c|}{ Planting method (B): } \\
\hline Ridges & $4.58 \mathrm{a}$ & $5.75 \mathrm{a}$ & $4.56 \mathrm{a}$ & $4.48 \mathrm{a}$ \\
\hline Wide furrows & $4.17 \mathrm{~b}$ & $4.83 \mathrm{~b}$ & $2.68 \mathrm{~b}$ & $2.97 \mathrm{~b}$ \\
\hline F-test & $*$ & $* *$ & $* *$ & $* *$ \\
\hline $\begin{array}{l}\text { Planting density } \\
30 \text { plants } / \mathrm{m}^{2}\end{array}$ & $3.98 \mathrm{~b}$ & $4.21 \mathrm{c}$ & $2.55 \mathrm{c}$ & $1.98 \mathrm{c}$ \\
\hline 45 plants $/ \mathrm{m}^{2}$ & $4.35 \mathrm{ab}$ & $5.46 \mathrm{~b}$ & $3.65 \mathrm{~b}$ & $3.73 \mathrm{~b}$ \\
\hline 60 plants $/ \mathrm{m}^{2}$ & $4.79 \mathrm{a}$ & $6.21 \mathrm{a}$ & $4.67 \mathrm{a}$ & $5.46 \mathrm{a}$ \\
\hline F-test & $* *$ & $* *$ & $* *$ & $* *$ \\
\hline \multicolumn{5}{|l|}{ Interaction : } \\
\hline A X B & N.S & N.S & $*$ & N.S \\
\hline $\mathrm{AXC}$ & N.S & $*$ & $* *$ & $* *$ \\
\hline B X C & N.S & N.S & N.S & N.S \\
\hline A X B X C & N.S & ** & N.S & N.S \\
\hline
\end{tabular}

*, ** and N.S.: significant, highly and not significant, respectively. Means within the same column for each factor followed by the same letter are not significantly different, according to DMRT at 0.05 level.

For the effect of plant density on downy mildew and purple blotch disease percentage, the data in Table 12 reveal markedly increase in downy mildew and purple blotch percentage with the third plant population $\left(60\right.$ plants $\left./ \mathrm{m}^{2}\right)$ followed by the second plant density (45 plants/ $\mathrm{m}^{2}$ ). Meanwhile, the lowest downy mildew and purple blotch percentage in onion plants was obtained from the first plant density (30 plants/ $\mathrm{m}^{2}$ ) in 2014/15 and 2015/16 seasons. This can be attributed to the increased plants per meter in dense stands, which are known to increase the moisture between plants at high plant density leading to high percentage in downy mildew disease. Similar results were also reported by Maude (1990) and Schwartz \& Mohan (2008) who indicated that new infections occur with a film of water present on the leaves for at least 26$\mathrm{h}$ and temperature between 3 and $14^{\circ} \mathrm{C}$. Latency period up to the development of new sporulation lasts from 9 to 16 days.

With respect to the interaction between onion transplanting date, method and plant density, the data of Table 13 reveal that the highest values in downy mildew and purple blotch percentage were obtained, when onions were transplanted at $15^{\text {th }}$ December in ridges method with 60 plants $/ \mathrm{m}^{2}$. 
TABLE 13. Highest values of disease parameters percentage as affected by significant interaction among the experimental factors in 2014/15 and 2015/16 seasons.

\begin{tabular}{cccc}
\hline Variable & Season & Highest values & Treatment \\
\hline Downy mildew \% & $2015 / 16$ & 5.75 & $15^{\text {th }}$ December x Ridges \\
& $2015 / 16$ & 6.90 & $15^{\text {th }}$ December x 60 plants $/ \mathrm{m}^{2}$ \\
& $2014 / 15$ & 7.88 & $15^{\text {th }}$ December $\times 60$ plants $/ \mathrm{m}^{2}$ \\
Purple blotch \% & $2015 / 16$ & 6.85 & $15^{\text {th }}$ December x Ridges x 60 plants/ \\
& $2014 / 15$ & 8.50 & $\mathrm{~m}^{2}$ \\
\hline
\end{tabular}

\section{Economic evaluation}

The results of the partial budget analysis (Fig. 1) showed that transplanting onions at early date $\left(15^{\text {th }}\right.$ Dec.) resulted in the highest values cost of cultivation, gross and net return per fad and benefit-cost ratio followed by $1^{\text {st }}$ January date, while $15^{\text {th }}$ January recorded the lowest ones.

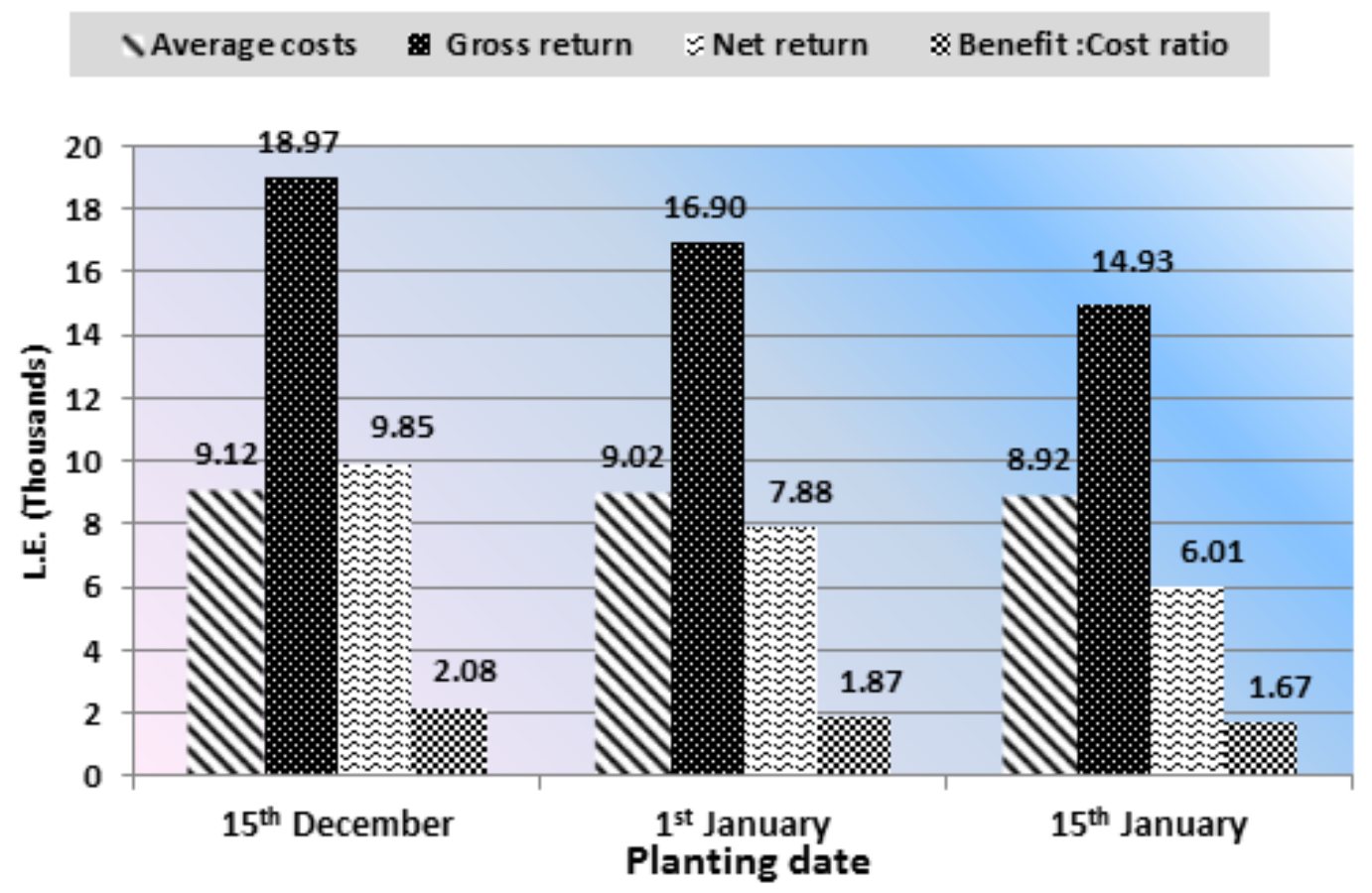

Fig.1. Average costs, gross return, net return and benefit: cost ratio (L.E./fad) of Giza Red onion yield as influenced by planting date as overall mean values through the two growing seasons.

Wide furrows planting method surpassed ridges planting method in the gross and net return per fad and benefit-cost ratio (Fig. 2). Concerning the effect of plant density, Fig. 3 showed that planting 45 plants per square meter gained the highest gross and net return per fad and benefitcost ratio followed by 60 and 30 plants $/ \mathrm{m}^{2}$, in order in 2014 /15 and 2015/16 seasons. 


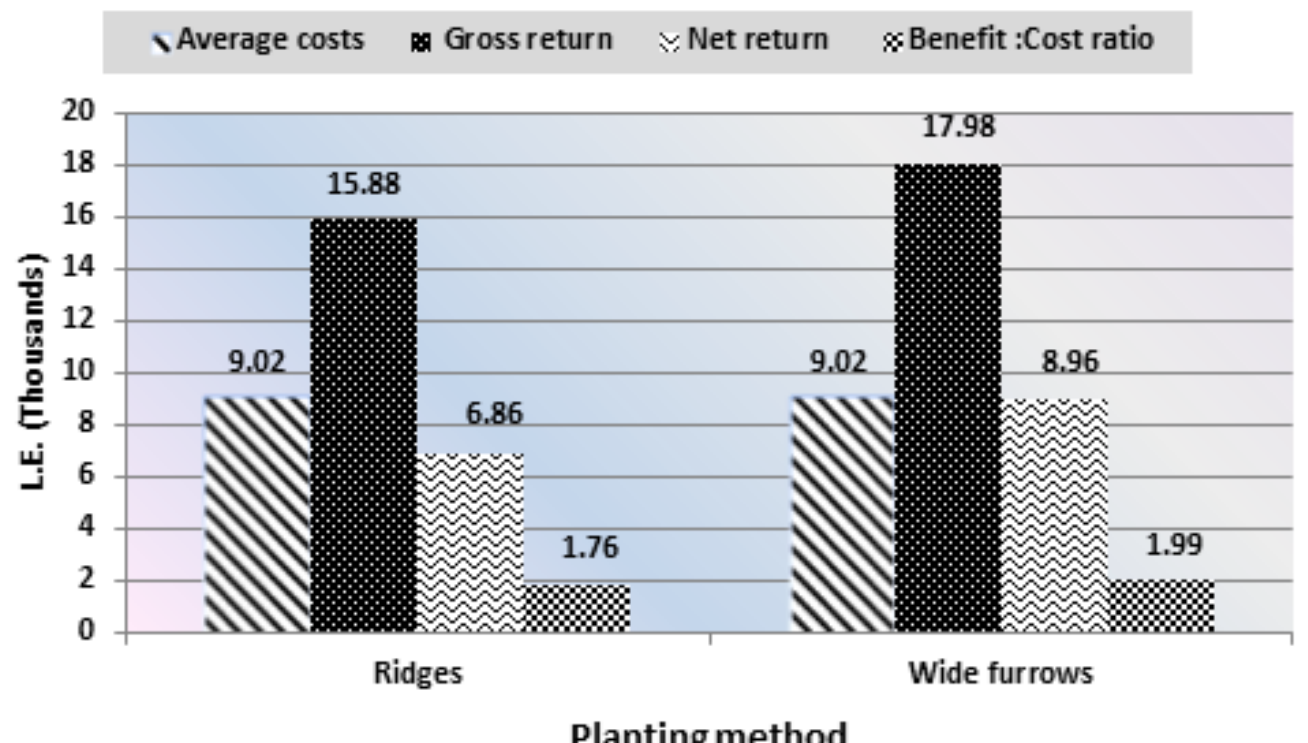

Fig. 2. Average costs, gross return, net return and benefit: cost ratio (L.E. /fad) of Giza Red onion yield as affected by planting method as overall mean values through the two growing seasons.

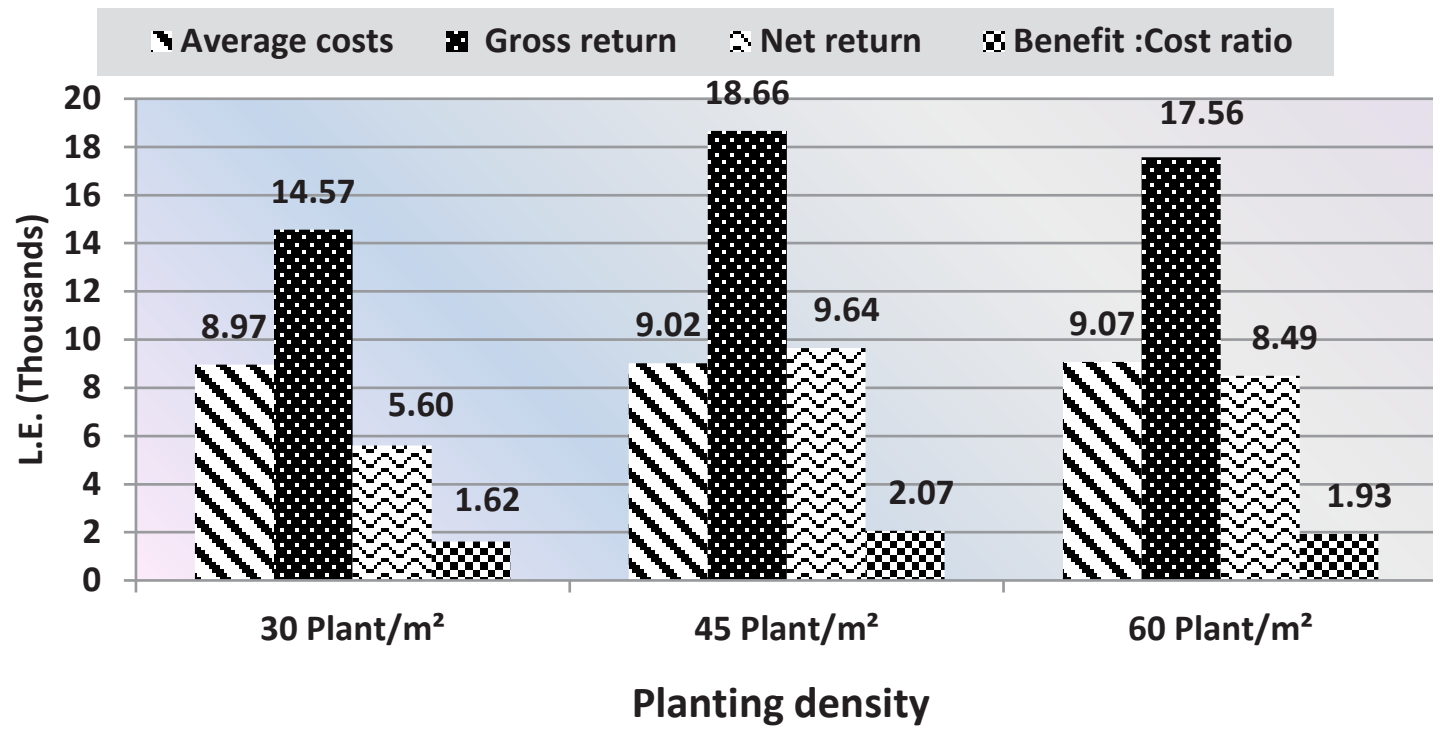

Fig. 3. Average costs, gross return, net return and benefit: cost ratio (L.E. /fed.) of Giza Red onion yield as influenced by plant density as overall mean values through the two growing seasons.

\section{Conclusions}

Finally, it can be concluded from the obtained results and from the economic point of view that transplanting onion plants at the early date $\left(15^{\text {th }}\right.$ December) with wide furrows planting method under the medium plant density (45 plants $/ \mathrm{m}^{2}$ ) could be recommended for the highest values of bulb yield and quality with optimum diseases control under the environmental conditions of North Delta area.

\section{References}

Ahmed, M.K. and Hassan, M.S.(1978) Effects of land prepration and planting method on onion (Allium cepa L.) production in the Sudan Gezira. Acta Hortic. 84, 27- 32. 
Al Abdulsalam, M.A. and Hamaiel, A.F. (2004) Effect of planting dates and compound fertilizers on growth, yield and quality of Hassawi onion under Al-Hassa oasis conditions. Sci. J. King Faisal Univ. (Basic and Applied Sciences), 5(1), 65- 79.

Ali, L., Hassan, M.W., Jamil, M., Iqbal, J., Yaqub, M.S., Akram, Ahmed, M., I. and Hussain, A. (2016 a) Effect of nursery bulb size and planting density on Thirps population, plant height and yield of onion (Phulkara variety) in Bahawalpur, Pakistan. Pak.J. Life Soc. Sci. 14(2), $96-103$.

Ali, M., Rab, A. ; Ali, J., Ahmad, H., Hayat, S., Wali, K., Rashid, A., Ahmad, W. and Muhammad, H. (2016 b) Influence of transplanting dates and population densities on the growth and yield of onion. Pure Appl. Biol. 5(2),345- 354.

Awas, G.; Abdisa, T., Tolessa, K. and Chali, A. (2010) Effect of intra - row spacing on yield of three onion (Allium cepa L.) varieties at Adami Tulu Agricultural Research Center (Mid Rift Valley of Ethiopia). $J$. Hortic. For. 2(1), 007- 011.

Bardisi, Samar A., El-Ghamriny, E.A., Gad, A.A. and Nawar, Dalia A.S. (2013) Bulbing ratio and dry weight of onion plant grown in sandy soil as affected by planting date, planting distance and potassium fertilizer rate.Zagazig J. Agric. Res. 40(3), 399 -410.

Bharti, N. and Ram, R.B. (2014) Estimating variation in the production, quality and economics of onion in response to transplanting dates and sulphur fertilization. European Academic Res. 2(4), 48314843-.

Bhatt, V.K., Patel, P.T., and Bhatt, R.K. (2007) Effect of methods and dates of sowing on yield of green onion in Kharif under North Gujarat conditions. Ind. J. Hort. 64(4), 475- 476.

Bosekeng, G. and Coetzer, G.M. (2015) Response of onion (Allium cepa L.) to sowing date and plant population in the Central Free State, South Africa. Afr. J. Agric. Res. 10(4), $179-187$.

Caruso, G., Conti, S., Villari, G., Borrelli, C., Melchionna, G., Minutolo, M., Russo, G. and Amalfitano, C. (2013) Effects of transplanting time and plant density on yield, quality and antioxidant content of onion (Allium cepa L.) in southern Italy. Sci. Horti. 166, 111- 120.

CIMMYT (1988) "From Agronomic Data to Farmer Recommendation: An Economic Work Book” D.F: pp. 31- 33 .

Cook, H.T. (1932) 'Downy Mildew Disease of Onion".N.Y. Agric. Exp. Station. Ithaca, New York. Mem. pp.140143.

Coolong, T.W. and Randle, W.M. (2003) Temperature influences flavor intensity and quality in 'Granex 33' onion. J. Amer. Soc. Hort. Sci. 128(2), 176- 181.

Corcoles, J.I., Dominguez, A., Moreno, M.A., Ortega J.F. and de Juan, J.A. (2015) A non - destructive methods for estimating onion leaf area. Irish J. Agric. Food Res. 54(1), $17-30$.

Develash, R. K. and Sugha, S. K. (1997) Incidence of downy mildew and its impact on yield. Indian Phytopathology, 50, 127-129.

Duncan, B. D. (1955) Multiple rang and multiple F-test Biometrics, 11, 1- 42.

Farrage, M.M. (1995) Influence of planting method and plant density on growth, yield and bulb quality of onion grown from sets. Assiut J. Agric. Sci. 26(1),73- 83.

George, E.B., Reid, L.T., Jeff, C., Cliff, R. and Hill, C.R. (2009) Sowing date, transplanting date, and variety effect on transplanted short - day onion production. Hort. Techn. 19(1), 66- 71.

Geries, L.S.M., Moursi, E.A. and Abo - Dahab, A.M.A. (2015) Effect of irrigation levels, cultivation methods and plant densities on productivity, quality of onion crop and some water relations in heavy clay soils. $J$. Soil Sci. and Agric. Eng., Mansoura Univ. 6(12), 1467 -1495 .

Gomez, K.A. and Gomez, A.A. (1984) "Statistical procedures for Agricultural Research". $2^{\text {nd }}$ ed. John Wiley and Sons, Inc. New York.

Griffiths, G., Trueman, L., Crowther, T., Thomas, B. and Smith, B. (2002) Onions a global benefit to health. Phytother Research, 16, 603 -615.

Haq, U.Z., Shah, A.H. and Malik, A.A. (2016) Effect of planting density on the yield of onion. J. Biology and Nature, 3(4), 139- 144.

Hatridge - Esh, K.A. and Bennett, J.P. (1980) Effects of seed weight, plant density and spacing on yield responses of onion. J. Hortic. Sci. 55(3), 247 -252.

Hoffmann, M. P., Petzoldt, C. H. and Frodsham, A. C. (1996) "Integrated Pest Management for Onions". New York State IPM Program Publication, No. 119., $78 \mathrm{p}$.

Ibrahim, N.D. and Adesiyun, A.A (2009) Effects of staggered planting dates on the control of Thirps tabaci Lindeman and yield of onion in Nigeria. Afr. J. Agric. Res. 4(1), $033-039$.

Islam, M.R., Mukherjee, A., Quddus, K.G., Sardar, P.K. and Hossain, M. (2015) Effect of spacing and fertilizer on the growth and yield of onion.Int. J. Sci. Tech. Res. 4(10), $308-312$. 
Jackson, M.L. (1967) "Soil Chemical Analysis". Prentice - Hall of India, Print Limited, New Delhi, pp: 115 .

Kandil, A.A., Sharief, A.E. and Fathalla, F.H. (2013) Effect of transplanting dates of some onion cultivars on vegetative growth, bulb yield its quality.Esci J. Crop Prod. 02(03), 72 -82.

Kanwar, M.S. and Akbar, P.I. (2013) Effect of planting methods on performance of onion varieties under cold desert conditions. The Bioscan, 8(3), 911 -913 .

Krauthausen, H. J., Richter, E. , Hagner, S. and Hommes, M. (2001) Epidemiology and control (based on thresholds) of leaf diseases (Peronospora destructor, Botrytis spp.) and thrips (Thrips tabaci) in onion. Acta Horticulturae, 25, 137-140.

Leilah, A.A., El-kalla, S.A., Mostafa, A.K. and Afifi, H.M.A. (2003) Performance of some local Egyptian onions strains under different planting dates. Sci. J. King Faisal Univ. (Basic and Applied Sciences), 4(1), 119- 136.

Mallor, C., Balcells, M., Mallor, F. and Sales, E. (2011) Genetic variation for bulb size, soluble solids content and pungency in the Spanish sweet onion variety Fuentes de Ebro.Response to selection for low pungency. Plant Breeding,130, 55- 59.

Maude, R. B. (1990) Leaf diseases of onions.In: "Onion and Allied Crops" H. D. Rabinowitch \& J. L. Brewster (Ed.), pp. 173-212. Boca Raton: CRC .

Mcgeary, D.J. (1985) The effects of plant density on the shape, size, uniformity, soluble solids content and yield of onions suitable for pickling. J. Hort. Sci.60(1), $83-87$.

Misra, A.D.D., Kumar, A. and Meitei, W.I. (2014) Effect of spacing and planting time on growth and yield of onion var. N-53 under Manipur Himalayas. Indian J. Hort. 71(2), 207 - 210.

MoslehUd-Deen, M.D. (2008) Effect of mother bulb size and planting time on growth, bulb and seed yield of onion. Bangladesh J. Agri. Res. 33(3),531537-.

Mostafizur, A.B.M. (2013) Performance studies on twenty seven onion Accessions under different planting date. M.Sc. Thesis, Bangladesh Agric. Univ., Bangladesh.

Nawer, F.R. (2006) Effect of ridges width and plant density of onion on yield of intercropped sugar beet and onion. J. Agric. Sci. Mansoura Univ. 31(8), 4837 -4847 .
Nguthi, F.N. (1993) The effect of nitrogen fertilization and plant density on growth, yield, quality and shelf - life of bulb onions (Allium cepa L.).M.Sc. Thesis, Univ., Nairobi, Kenya.

Pablo, H. Gonzalez and Colnago, P. (2011)Quantitative studies on downy mildew (Peronospora destructor Berk. Casp.) affecting onion seed production in southern Uruguay. Eur. J. Plant Pathol. 129, $303-$ 314.

Piper, C.S. (1950) "Soil and Plant Analysis". Interscience Publishers Inc., New York.

Raziq, F., I. Alam, Naz, I. and Khan, H. (2008) Evaluation of fungicides for controlling downy mildew of onion under field conditions.Sarhad $J$. Agric. 24(1), 85- 92.

Rizk, Fatma A. (1997) Productivity of onion plant (Allium cepa L.) as affected by method of planting and NPK application. Egypt. J. Hort. 24(2), 219. 238.

Saud, S., Yajun, C., Razaq, M., Luquman, M., Fahad, S., Abdullah, M. and Sadiq, A. (2013) Effect of Potash levels and row spacings on onion yield. $J$. Biol. Agric. Health Care, 3(16), 118 - 127.

Schwartz, H. F. and Mohan, S. K. (2008) "Compendium of Onion and Garlic Diseases". St. Paul: APS. 127p.

Shahanaz, E., Razdan, V. K and Raina, P. K. (2007) Survival, dispersal and management of foliar blight pathogen of onion. J. Mycol. Pl. Pathol. 37(2), 213 - 214.

Shock, C.C., Feibert, E.B.G., Riveira, A. and Saunders, L.D. (2015) Response of onion yield, grade, and financial return to plant population and irrigation system. Hort Science, 50(9), 1312- 1318.

Siliquini, O.A., Orioli, G.A. and Lobartini, J.C. (2015) Onion yield as affected by plant density, nitrogen level and loss of leaf area. $\phi Y T O N, \mathbf{8 4}, 338-344$.

Singh, A.K., Singh, V. and Singh, V.K. (2001) Effect of set size, distance and date of planting on growth and bulb yield of Kharif onion (Allium cepa L.). J. Farming Sys. Res., Develop. 7(1), 72- 76.

Surviliene, E., Valiuskaite, A. and Raudonis, L. (2008)The effect of fungicides on the development of downy mildew of onions.ZemdirbysteAgriculture, 95 (30), 171-179.

Van Doorn, A. M. (1959) Onderzoekingen over het optreden en de bestrijding van valsemeeldauw (Peronospora destructor) bijuien. TijdschrivenPlantenziekten, 65, 193-255. 
Viranyi, F. (1974) Downy mildew of onion. Novenyvdlem, 10, 205- 209.

Yadav, P.M., Rakholiya, K. B. and Pawar, D. M. (2013) Evaluation of bioagents for management of the onion purple blotch and bulb yield loss assessment under field conditions. The Bioscan, 8 (4), 1295- 1298.

Zubelidia, A. and Gases, J. (1977) Effect of spacing and number of stem on the earliness and total yield of Tomato cultivars.Product. Veg. 7, 73- 80.

\section{إستجابة إنتاجية وجودة البصل لمواعيد وطرق زراعة وكثافات نباتية مختلفة

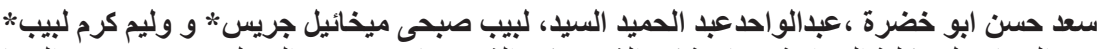

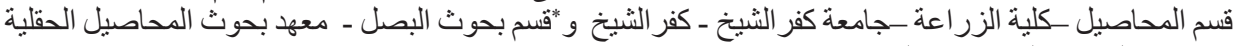 - مركز البحوث الزراعية ـ الجيزة- مصر.}

أجريت تجربة حقلية بمحطة البحوث الزراعية بسخا - محافظة كفرالثيخ - خلال موسمى النمو

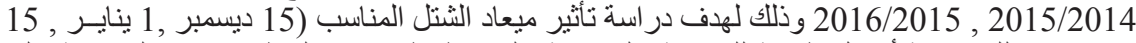

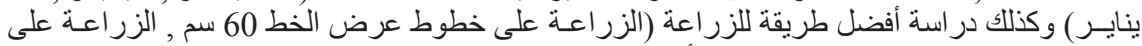

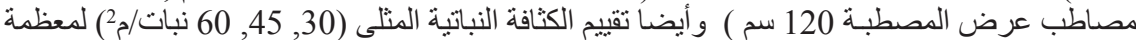
النمو الخضرى و المحصول و الجودة للبصل وذللك تحت ظروف الته منطقة شمال الدلتا. هذا ويمكن تلخيص أهم نتائج الدرل اسة فيما يلى:

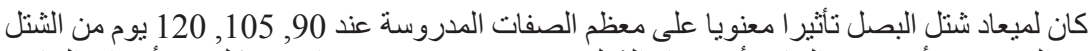

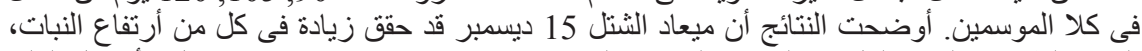

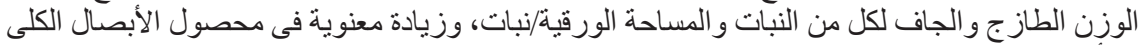

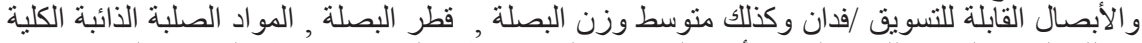

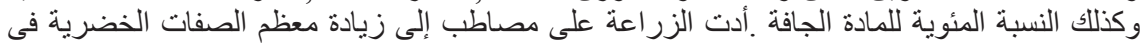

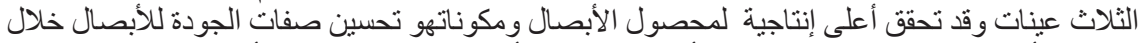

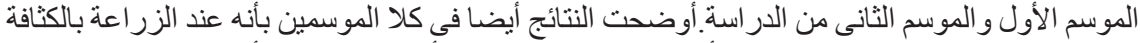

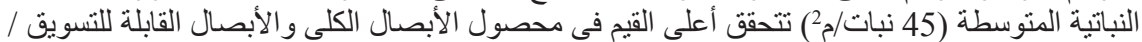
فدان خلال موسمى2015/2014 2016/2015.

أوضحت النتائج أن ميعاد شتل البصل فى 15 ديسمبر على مصاطب أدى إلى زيادة معنوية لأرتفاع

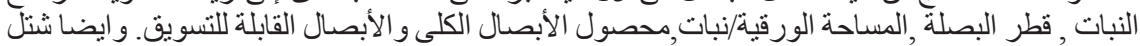

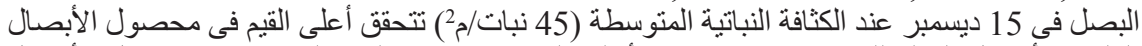

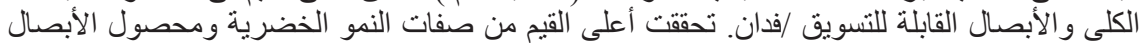
الكلى و الأبصال القابلة للتسويق و تحسين صفات الجودة للألأبصال عند الزر اعة العلى مصاطب بكثنافة نباتية 45 نبات/2مات.

أظهرت القيمة الإقتصادية بأن أعلى زيادة فى إجمالى العائد وكذلك صافى العائد قد تحقق بشتل البصل

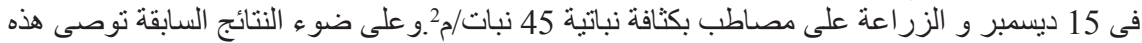

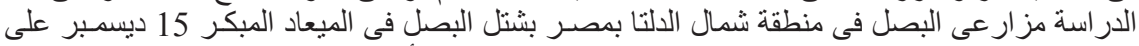

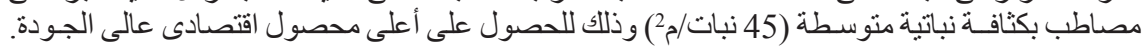

